\section{A computer program to generate signal-detection theory values for sensitivity and response bias}

\author{
RICK M. GARDNER and RUSSEL BOICE \\ University of Southern Colorado, Pueblo, Colorado
}

The theory of signal detection was originally proposed by Tanner and Swets (1954) and has come to serve as an alternative to classical psychophysical methodologies. Signal detection's greatest advantage over classical psychophysical methodologies is the separate determination of parameters reflecting sensory and nonsensory factors.

The sensitivity of the decision-making process is a function of the variable $d^{\prime}$, which is equal to the distance between the noise and signal-plus-noise distribution and which is monotonically related to the signal strength. Nonsensory factors are reflected in the response criterion. The most commonly used measure of response criterion is $\beta$, which is defined as the ratio of the signal-plus-noise distribution at the criterion to the ordinate of the noise distribution. Gardner, Dalsing, Reyes, and Brake (1984) recently published a table of $\beta$ values corresponding to various hit and false-alarm rates.

Traditionally, researchers in psychophysics have been primarily interested in measures of sensory sensitivity. The matter of how a subject sets or maintains a criterion at the appropriate value and adjusts that value to take account of relevant intercurrent events has been largely ignored (Treisman \& Williams, 1984).

One disadvantage of $\beta$ as a measure of response criterion is that it forms an asymmetric distribution such that values cannot be easily averaged over subjects, trial blocks, and so forth. By taking the natural logarithm of $\beta$, one derives a measure of response criterion that can be averaged. In addition, $\ln (\beta)$ is invariant over sensitivity under maximization of expected value. The conversion to $\ln (\beta)$ is helpful when the researcher wishes to average $\beta$ or to do other parametric analyses of $\beta$ values.

We describe a program (see Appendix), written for the Apple computer, that generates measures of $d^{\prime}, \beta$ and $\ln (\beta)$ from a given pair of hit and false-alarm rates. The ZGEN program is written in Applesoft and uses an approximation for the inverse normal integral function, which is defined as the value of $X$ such that for a given $Q$ :

$$
Q=\int_{x}^{\infty} \frac{1}{2 \pi} e^{-t^{2} / 2} d t
$$

The following rationale approximation for this function is used:

This research was supported by Grant No. 5 SO6 RR08197, funded by the Division of Research Resources and the National Institute of Mental Health. Requests for reprints should be sent to R. M. Gardner, Department of Psychology, University of Southern Colorado, Pueblo, CO 81001 .

$$
X=t-\left(c_{0}+c_{1} t+c_{2} t^{2}\right) /\left(1+d_{1} t+d_{2} t^{2}+d_{3} t^{3}\right),
$$

where $t=\sqrt{\ln \left[1 /\left(Q^{2}\right)\right]}, c_{0}=2.515517, d_{1}=1.432788$, $c_{1}=.802853, d_{2}=.189269, c_{2}=.010328$, and $d_{3}=$ .001308 . This approximation is valid for $0<Q<0.5$ and is accurate to $\pm 4.5 \times 10^{-4}$. Brophy (1985) describes in detail procedures for other approximations of the inverse normal distribution function.

The present program breaks the function down to numerator and denominator sections and then expands the approximation, using the symmetry of the function to allow values ranging between 0 and 1 . The expanded function called $\mathrm{FN} \mathrm{Z}$ in the program, along with the function FN G (the standardized normal distribution curve), can then be used to calculate the desired quantities. The FN R function is used to round the resulting values to three decimal places.

An additional feature of this program is that it calculates values of $d^{\prime},(\beta)$, and $\ln (\beta)$, even if individual subjects' hit or false-alarm rates contain proportions of 0 or 1 , which correspond to infinite $d^{\prime}$ s. In these circumstances, the program follows the procedure outlined by Macmillan and Kaplan (1985), in which 0 is replaced by $1 /(2 N)$ and 1 is replaced by $1-1 /(2 N)$, where $N$ is the number of trials on which the relevant stimulus was presented. When a 0 or 1 is entered as a data point, the program requests $N$. The resulting output is marked by an asterisk as a reminder that this procedure was used for those values.

An advantage of the program is that it allows computation of sensitivity and response bias values with greater accuracy than do existing tables of those values. Hit and false-alarm proportions of three-decimal accuracy may be entered, and all outputs are also to three decimals. In addition, the program calculates $\ln (\beta)$, which should prove helpful to researchers who wish to average response bias values across trials or across subjects. This program should prove helpful to individuals wishing to rapidly calculate measures of sensitivity and response bias where the signal detection paradigm has been followed.

\section{REFERENCES}

BROPHY, A. L. (1985). Approximation of the inverse normal distribution function. Behavior Research Methods, Instruments, \& Computers, 17, 415-417.

Gardner, R. M., Dalsing, S., Reyes, B., \& Brake, S. (1984). Table of criterion values $(\beta)$ used in signal detection theory. Behavior Research Methods, Instruments, \& Computers, 16, 425-436.

Macmillan, N. A., \& KaPlan, H. L. (1985). Detection theory analysis of group data: Estimating sensitivity from average hit and falsealarm rates. Psychological Bulletin, 98, 185-199.

TANNER, W. P., \& SWETS, J. A. (1954). A decision-making theory of visual detection. Psychological Review, 61, 401-409.

Treisman, M., \& Williams, T. C. (1984). A theory of criterion setting with an application to sequential dependencies. Psychological Review, 91, 68-111. 


\section{APPENDIX}

Program to Calculate Signal Detection Values of $d^{\prime}, \beta$, and $\ln (\beta)$.

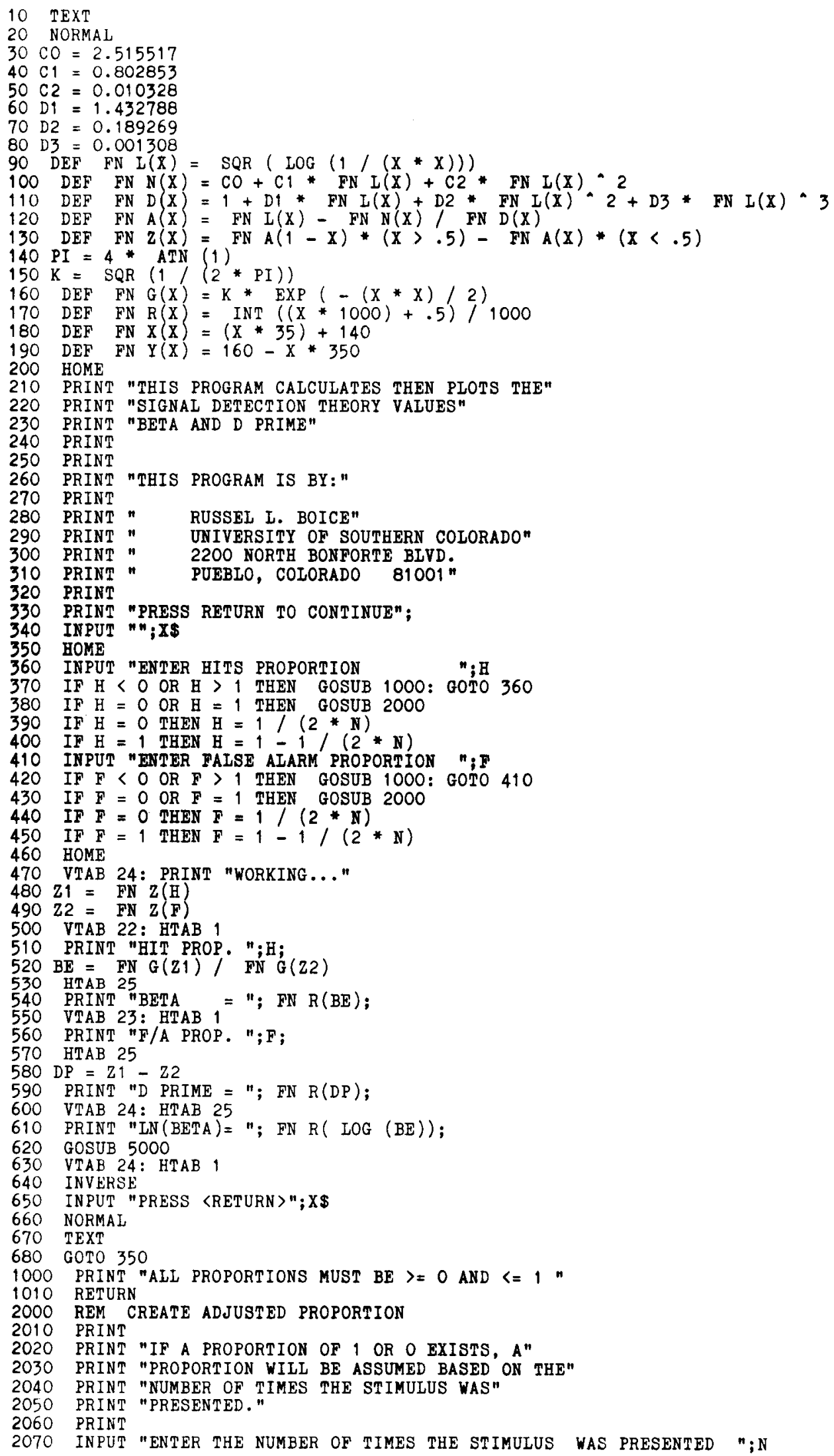


APPENDIX (continued)

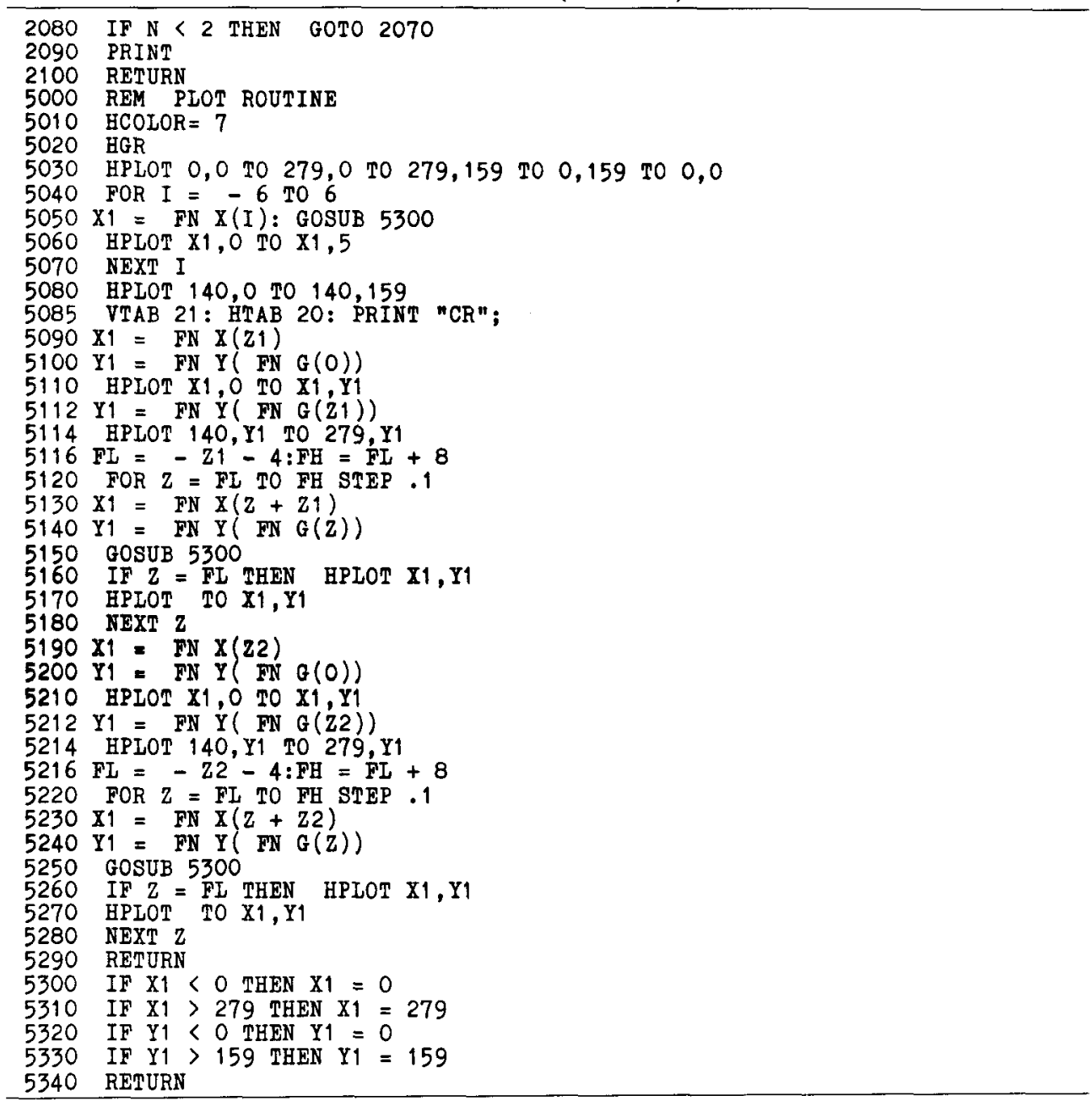

(Revision accepted for publication January 27, 1986.) 